\title{
ÍNDICES DE TRAUMA
}

\author{
TRAUMA SEVERITY INDICES
}

Gerson Alves Pereira Júnior ${ }^{1}$; Sandro Scarpelini'; Aníbal Basile-Filho² \& José Ivan de Andrade ${ }^{3}$

\begin{abstract}
'Médicos Assistentes da Unidade de Emergência do Hospital das Clínicas; ${ }^{2}$ Docente do Departamento de Cirurgia, Ortopedia e Traumatologia e Chefe da Disciplina de Terapia Intensiva; ${ }^{3}$ Docente do Departamento de Cirurgia, Ortopedia e Traumatologia. Faculdade de Medicina de Ribeirão Preto (FMRP) da Universidade de São Paulo (USP).

CorResPondência: Dr. Gerson Alves Pereira Júnior - Rua Iguape, 747 apto 12 - C - Jardim Paulista - CEP 14090-000Ribeirão Preto - SP (e-mail: gersonapj@netsite.com.br)
\end{abstract}

PEREIRA Jr. GA; SCARPELINI S; BASILE-FILHO A \& ANDRADE JI. Índices de trauma. Medicina, Ribeirão Preto, 32: 237-250, jul./set. 1999.

RESUMO: A caracterização e documentação dos vários graus de lesões traumáticas são os requisitos básicos para a avaliação do sistema de atendimento ao trauma e para o desenvolvimento de iniciativas para o seu controle. Os índices de trauma são valores matemáticos ou estatísticos, quantificados por escores numéricos, que variam de acordo com a intensidade e os tipos de lesões decorrentes do traumatismo, medidos através de parâmetros anatômicos e fisiológicos. Tais índices representam uma importante e essencial ferramenta para estudos clínicos em trauma. Este artigo descreve vários dos índices de trauma mais comumente utilizados e suas aplicações.

UNITERMOS: Índices de Gravidade do Trauma. Escala de Gravidade do Ferimento. Escala Resumida de Ferimentos. Escala de Coma de Glasgow. Mortalidade.

\section{INTRODUÇÃO}

A qualidade do atendimento ao traumatizado depende da ação de todos os profissionais envolvidos nesta missão, começando pelo adequado atendimento na cena do acidente, transporte rápido e seguro (por via terrestre ou aérea) com comunicação prévia ao serviço que irá recebê-lo, continuando por uma competente avaliação, ressuscitação volêmica, acurado diagnóstico, adequado tratamento intra-hospitalar, e finalizando por uma reabilitação que o integre novamente à sociedade, mantendo ao máximo a sua capacidade de trabalho, ou, ao menos, permitindo-lhe condições de uma sobrevida digna.

A qualidade do atendimento depende, também, de medidas administrativas (desde recursos humanos, habilitados em educação continuada, até recursos diagnósticos e terapêuticos modernos e disponíveis) e de planejamento do atendimento (estratificação dos hospitais para o atendimento de pacientes cuja gravidade do quadro esteja dentro da sua capacidade, em termos de recursos humanos e de diagnóstico e tratamento) sendo cada etapa fundamental, influenciando na morte ou sobrevida do paciente.

Os traumas podem variar desde escoriações superficiais, sem nenhum risco de vida, até grandes contusões ou ferimentos graves que causam a morte instantânea da vítima. Assim, o conhecimento do nível de gravidade de um paciente traumatizado é fator decisivo para orientar não apenas a conduta e o nível de complexidade hospitalar exigido, como também para aferir a qualidade do atendimento prestado.

Os índices de trauma são sistemas de pontuação criados para avaliar as alterações fisiológicas, a gravidade das lesões anatômicas e a probabilidade de sobrevida dos pacientes politraumatizados ${ }^{(1)}$. 
A adequada definição da gravidade do quadro clínico do paciente permite a comparação de diversas modalidades terapêuticas entre lesões de gravidade equivalentes, comparação de resultados dentro de um mesmo serviço ou entre serviços diferentes, o que, em última análise, avalia a qualidade do atendimento prestado, podendo identificar e alterar condutas cujos resultados não sejam comparáveis aos resultados internacionalmente aceitos. A análise dos índices de trauma permite, ainda, a apreciação de vários outros objetivos (Tabela I).

\section{Tabela I - Índices de trauma: objetivos}

- Quantificação de alterações fisiológicas

- Quantificação das lesões anatômicas

- Cálculo da probabilidade de sobrevida

- Triagem de pacientes politraumatizados para centros de trauma

- Pesquisa clínica

- Avaliação de resultados institucionais

- Controle de qualidade

- Epidemiologia

- Campanha de prevenção de violência

- Pagamento de despesas médico-hospitalares

No politraumatizado, a adequada definição da gravidade infere na probabilidade de sobrevida para o paciente, o que compromete o médico responsável pelo atendimento por um resultado até certo ponto previsível, baseado no escore de gravidade. Isto é importante, pois evita um pensamento comum do médico que atende traumatizados, que é não se sentir pressionado por resultados, já que considera "lucro" conseguir fazer algo para o politraumatizado.

Os fatores que influenciam na morbidade ${ }^{(2)}$ são:

- atrasos no diagnóstico (após 24 horas da admissão).

- erros no diagnóstico (lesões despercebidas devido a inadequado exame físico ou má interpretação de exames diagnósticos complementares).

- erros de julgamento (decisões diagnósticas ou terapêuticas feitas contrariamente aos dados disponíveis).

- erros de técnica (que ocorrem durante a realização de procedimentos diagnósticos ou terapêuticos).

- doença do paciente (complicações inevitáveis, devido a progressão de doença subjacente).

As mortes são classificadas em ${ }^{(2)}$ :

- inevitáveis (lesão anatômica ou combinação de lesões consideradas de sobrevivência impossível, mesmo com tratamento adequado e a tempo, ge- ralmente, correspondem a uma probabilidade de sobrevida, de acordo com os índices de trauma, menor que $25 \%$ );

- potencialmente salváveis (lesão anatômica ou combinação de lesões consideradas muito graves, mas com sobrevivência possível dentro de condições ótimas de atendimento; geralmente, a probabilidade de sobrevida encontra-se entre 25 e $50 \%$ );

- francamente evitáveis (lesão anatômica ou combinação de lesões consideradas de sobrevida possível, com probabilidade de sobrevida maior que 50\%).

Nos EUA, foi delineado o Major Trauma Outcome Study (MTOS) ${ }^{(3)}$, que é um levantamento descritivo e retrospectivo dos índices de trauma e do resultado em termos de sobrevida, coordenado pelo Comitê de Trauma do Colégio Americano de Cirurgiões entre os anos de 1982 e 1987, compreendendo dados de 80.544 pacientes politraumatizados e de 139 hospitais, consistindo a base para comparação de resultados $^{(3)}$.

Na Unidade de Emergência, a Disciplina de Cirurgia do Trauma tem se preocupado com a utilização dos índices de gravidade, para avaliar a qualidade do serviço prestado e a comparação dos resultados com os dos maiores centros do país e do exterior. Para tanto, há cinco anos emprega a avaliação do Revisited Trauma Score (RTS).

Para complementar a avaliação da gravidade dos pacientes politraumatizados, iniciou-se, a partir de 1996, a utilização de outro índice, o Injury Severity Score (ISS), que, em associação ao RTS, forma a classificação denominada TRISS, a qual permite estabelecer a probabilidade de sobrevida do paciente, podendo-se avaliar a qualidade do serviço e fazer comparação entre eles.

A seguir, é explicado objetivamente o modo de obtenção de cada um deles, o que permitirá seu cálculo, sem dificuldades, pelas equipes de residentes e graduandos, em estágio ou plantão, pela Cirurgia da Unidade de Emergência.

$\mathrm{Na}$ descrição dos índices de trauma, as abreviações da nomenclatura em inglês serão mantidas, pois, assim, eles são conhecidos e citados na literatura médica.

\section{REVISITED TRAUMA SCORE (RTS)}

O RTS é um índice classificado como fisiológico, por levar em consideração os parâmetros das funções vitais do paciente ${ }^{(4)}$ Surgiu como uma evolução do Trauma Score $(\mathrm{TS})^{(5)}$, criado em 1981, porém sem 
a necessidade de avaliação do enchimento capilar e esforço respiratório ${ }^{(4)}$.

É proveniente da avaliação do estado neurológico pela Escala de Coma de Glasgow - E.C.G. (Tabela II), da pressão arterial sistólica (PAS) e da frequência respiratória (FR). As variáveis variam de 4 (normal) a 0 (Tabela III).

É um índice largamente utilizado na triagem préhospitalar, onde é chamado de t-RTS, sendo seus valores simplesmente somados e, de acordo com o valor total (que varia de 0 a 12), o paciente é transportado para determinado centro de trauma, previamente classificado, de acordo com a capacidade dos seus recursos diagnósticos, terapêuticos e humanos.

Para a avaliação intra-hospitalar, os valores das variáveis devem ser ponderados e somados:

\section{RTS = 0,9368 $\times$ E.C.G. $_{v}+\mathbf{0 , 7 3 2 6} \times$ PAS $_{v}+0,2908 \times$ FR $_{v}$}

onde v é o valor (de 0 a 4) correspondente as variáveis medidas na admissão do paciente.

Dessa maneira, o RTS poderá variar de 0 a aproximadamente 8, permitindo frações. Quanto maior o valor, melhor será o prognóstico, sendo possível o conhecimento da probabilidade de sobrevida. (Tabela IV).

Para exemplificar a utilização do RTS, um paciente vítima de atropelamento que dá entrada no serviço de emergência, consciente, orientado (E.C.G = 15), com PA = 150x100 mmHg, FC = 110 bpm e FR $=34 \mathrm{ipm}$, tem atribuído a essas variáveis, pela Tabela III, os seguintes valores:

$$
\begin{aligned}
& \text { E.C.G }=4 \\
& \text { PAS }_{v}=4 \\
& \text { FR }_{v}=3
\end{aligned}
$$

A seguir, com o auxílio da calculadora, equaciona-se a fórmula do RTS:

$$
\text { RTS }=0.9368 \times 4+0,7326 \times 4+0,2908 \times 3=7,55
$$

Com o valor obtido e o uso da Tabela IV, chegamos à probabilidade de sobrevida (Ps) desta vítima, que varia de 96,9 a 98,8\%

Como observado, o cálculo do RTS é extremamente simples, não dependendo da utilização de fórmulas ou dados complexos, nem mesmo de exames subsidiários. Seu cálculo deve ser feito na admissão do paciente.

\begin{tabular}{|ll|}
\hline \multicolumn{2}{|c|}{ Tabela II - Escores da Escala de Coma de Glasgow } \\
\hline - -espontânea & 4 \\
- -ao comando verbal & 3 \\
- -à dor & 2 \\
- -nenhuma & 1 \\
Resposta verbal & \\
\hline - orientado & 5 \\
- confuso & 4 \\
- palavras inapropriadas & 3 \\
- sons incompreensíveis & 2 \\
- nenhuma & 1 \\
Melhor resposta motora & \\
\hline - obedece aos comandos & 6 \\
- localiza a dor & 5 \\
- retirada a dor & 4 \\
- descorticação & 3 \\
- descerebração & 2 \\
- nenhuma & 1 \\
\hline
\end{tabular}

\begin{tabular}{|cccccc|}
\hline \multicolumn{6}{|l|}{ Tabela III - Escores do RTS para cada parâmetro } \\
\hline $\begin{array}{c}\text { Escala de Coma } \\
\text { de Glasqow }\end{array}$ & Valor & $\begin{array}{c}\text { Pressão } \\
\text { Sistólica } \\
\text { (mmHq) }\end{array}$ & Valor & $\begin{array}{c}\text { Freqüência } \\
\text { Respiratória } \\
\text { (ipm) }\end{array}$ & Valor \\
\hline $13-15$ & 4 & $>89$ & 4 & $10-29$ & 4 \\
$9-12$ & 3 & $76-89$ & 3 & $>29$ & 3 \\
$6-8$ & 2 & $50-75$ & 2 & $6-9$ & 2 \\
$4-5$ & 1 & $1-49$ & 1 & $1-5$ & 1 \\
3 & 0 & 0 & 0 & 0 & 0 \\
\hline
\end{tabular}

\begin{tabular}{cc}
$\begin{array}{l}\text { Tabela IV - Probabilidade de sobrevida para RTS } \\
\text { de números inteiros }\end{array}$ \\
\hline RTS & Probabilidade de sobrevida (PS) \\
\hline 8 & 0,988 \\
7 & 0,969 \\
6 & 0,919 \\
5 & 0,807 \\
4 & 0,605 \\
3 & 0,361 \\
2 & 0,172 \\
1 & 0,071 \\
0 & 0,027 \\
\hline
\end{tabular}




\section{INJURY SEVERITY SCORE (ISS)}

O ISS é um índice de gravidade, classificado como anatômico, por levar em consideração as lesões provocadas nos vários segmentos do corpo ${ }^{(6)}$. A gravidade das lesões anatômicas é determinada através do exame físico, testes radiológicos, cirurgia e autópsia ${ }^{(7)}$.

Seu desenvolvimento foi baseado no Abreviated Injury Scale (AIS) ${ }^{(8)}$. O AIS é uma lista de milhares de lesões, cada uma com valor de gravidade variando de 1 a 6 , que tem sido submetido a revisões desde 1971. O organismo é dividido, para efeito de índice, em seis (06) regiões: 1) cabeça e pescoço, 2) face, 3) tórax, 4) abdome/conteúdo pélvico, 5) extremidades e anel pélvico e 6) geral ou externo ${ }^{(8)}$. A Tabela $V$ é um resumo do AIS, utilizado pelo Toronto General Hospital, e pretende facilitar a classificação das lesões, já que a lista total do AIS possui mais de trinta e seis (36) páginas.

As lesões são classificadas em: 1) leve, 2) moderada, 3) grave, sem risco iminente de vida, 4) grave, com risco iminente de vida, 5) crítica, de sobrevida duvidosa e 6) quase sempre fatal.

O ISS é calculado após a classificação dos índices mais graves de cada uma das seis (06) regiões, escolhendo-se os três (03) valores das AIS mais altos em segmentos corpóreos diferentes e realizando-se a soma dos quadrados desses índices. Desta maneira, o ISS pode variar de 1 a 75 pontos. Qualquer paciente com uma lesão AIS - 6, tem um ISS = 75, por definição.

Como exemplo, imaginemos uma vítima de queda de cinco (05) metros de altura com fratura de 5 arcos costais, contusão pulmonar leve e trauma esplênico grave, que tem, pela Tabela $\mathrm{V}$, a seguinte avaliação:

$$
\begin{aligned}
\text { Cabeça/pescoço } & =1 \\
\text { Face } & =1 \\
\text { Tórax } & =3 \\
\text { Abdome } & =4 \\
\text { Extremidades } & =1 \\
\text { Geral } & =1 \\
\text { ISS }=4^{2}+3^{2}+1^{2} & =26
\end{aligned}
$$

Um ISS de 16 é preditivo de mortalidade em torno de $10 \%$ e define o trauma como sendo grave, em termos de lesão anatômica ${ }^{(9)}$.

Em função de suas características, esses índices não podem ser usados para fins de triagem, nem para determinação do prognóstico à admissão. Seu cálculo é feito após exames radiológicos, cirurgias ou mesmo autópsia.

O ISS correlaciona-se com a mortalidade, mas tem limitações, uma vez que incorpora apenas os maiores valores do AIS de cada região do corpo e das três maiores, considerando que lesões com o mesmo valor do AIS têm a mesma gravidade, não interessando a importância daquela região do $\operatorname{corpo}^{(9)}$. Como resultado, alguns valores do ISS contêm dados de pacientes com lesões heterogêneas, que têm prognósticos em termos de sobrevida/morte substancialmente diferentes, com redução do poder de predição de resultados $^{(9)}$.

O ISS correlaciona-se mais com a mortalidade tardia, enquanto que o RTS correlaciona-se melhor com a mortalidade precoce.

\section{PERFIL ANATÔMICO - ANATOMIC PROFILE (AP)}

As limitações do ISS, a crescente necessidade por maior precisão na quantificação das lesões e a necessidade de comparar grupos de pacientes com lesões similares motivaram o desenvolvimento de uma descrição de lesões de quatro valores (A, B, C e D), o Anatomic Profile ${ }^{(10)}$. Os primeiros três (03) valores resumem todas as lesões de maior gravidade (AIS $>2$ ) da cabeça/cérebro e coluna espinhal (A), tórax e região frontal do pescoço (B) e todas as demais lesões sérias (C). D é o resumo de todas as lesões não graves (AIS - 1 ou 2). A Tabela VI mostra os componentes deste índice.

A primazia das lesões cranianas e do tórax, como fonte de mortalidade, foi o que levou a essa associação de lesões dos componentes AP.

Os valores componentes do AP são a raiz quadrada da soma dos quadrados dos escores do AIS para todas as lesões associadas. Este método leva a uma diminuição da contribuição de lesões outras que as mais severas, em uma área particular do corpo (Tabela VII).

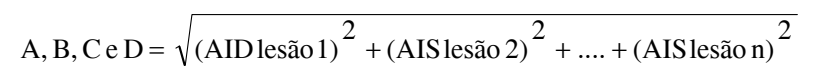

O AP proporciona uma descrição mais precisa da lesão anatômica do que o ISS, pois considera todas as lesões com AIS > 2 dentro de uma mesma região ou segmento corpóreo. Isto aumenta a acurácia na avaliação da qualidade do serviço e pode ser usado efetivamente para descrever a gravidade do quadro clínico do paciente e predizer vários resultados, tais como a sobrevida/morte, incapacidade e tempo de internação hospitalar/CTI.

Em função da grande utilização do ISS, em quase todos os centros de trauma do mundo, ainda é necessário testar a validade do AP em grandes casuísticas. 


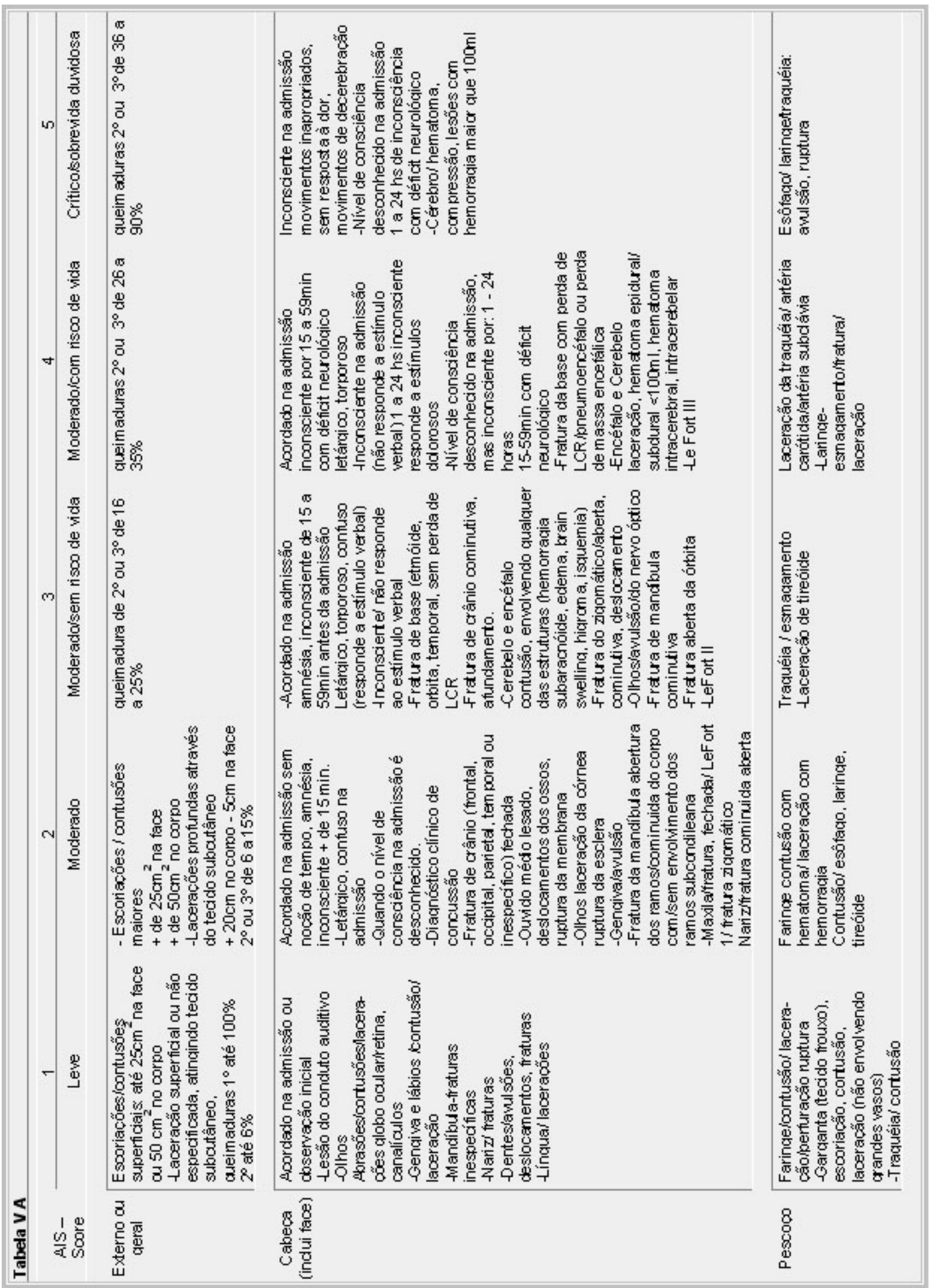




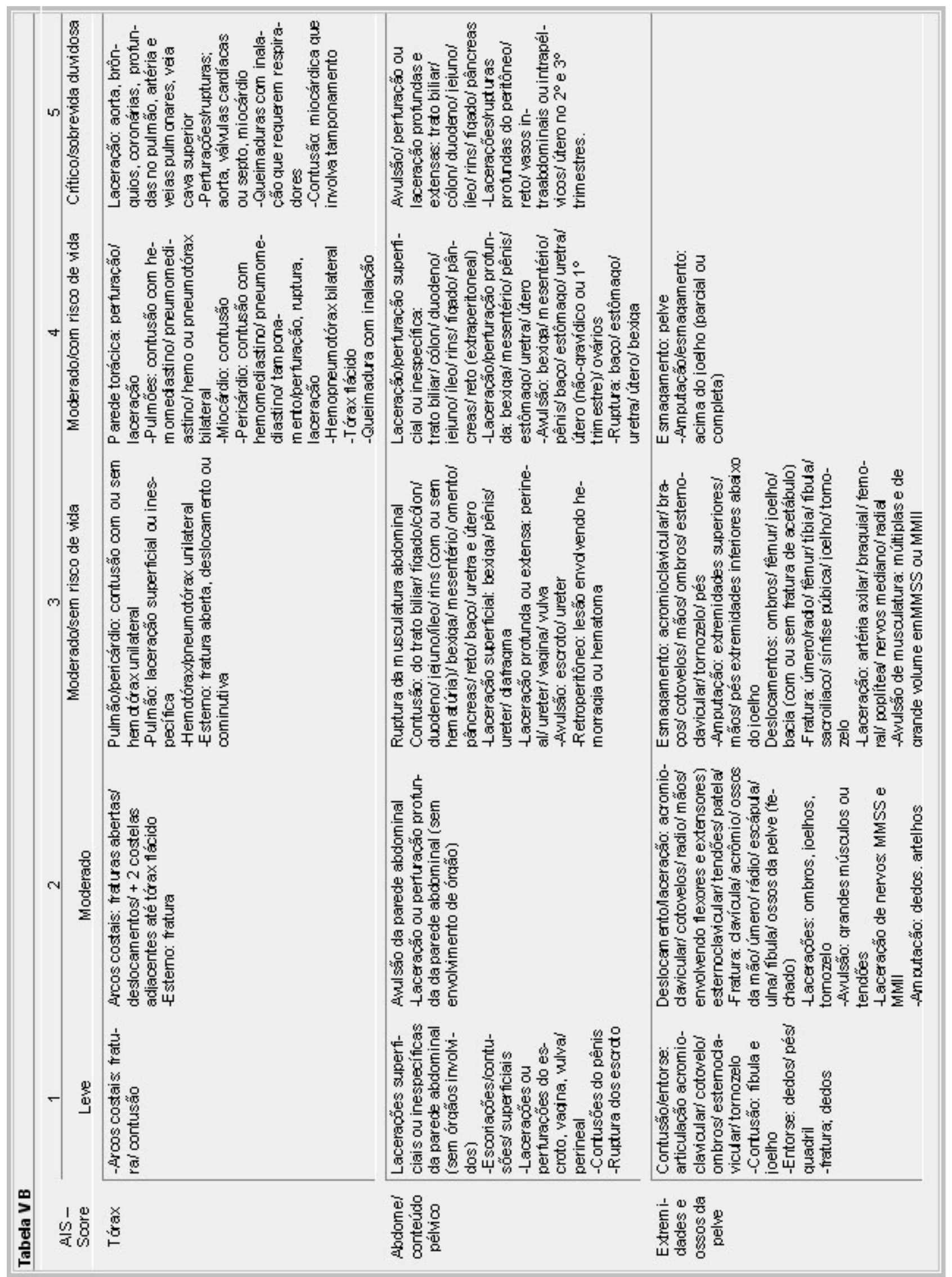




\begin{tabular}{|c|c|c|c|}
\hline \multicolumn{4}{|c|}{ Tabela VI - Componentes do AP } \\
\hline Componente & Lesão & $\begin{array}{c}\text { Classificacão } \\
\text { AIS }\end{array}$ & $\begin{array}{l}\text { Reqião do } \\
\text { corpo }\end{array}$ \\
\hline A & $\begin{array}{l}\text { Crânio/Cér ebro } \\
\text { Medula espinhal }\end{array}$ & $\begin{array}{l}3-5 \\
3-5\end{array}$ & $\begin{array}{c}1 \\
1,3,4\end{array}$ \\
\hline $\mathrm{B}$ & $\begin{array}{l}\text { Tórax } \\
\text { Pescoço anterior }\end{array}$ & $\begin{array}{l}3-5 \\
3-5\end{array}$ & $\begin{array}{l}3 \\
1\end{array}$ \\
\hline C & $\begin{array}{l}\text { Abdome/pelve } \\
\text { Coluna (sem medula) } \\
\text { Fratura pélvica } \\
\text { Artéria femoral } \\
\text { Esmaqamento (perna) } \\
\text { Amputação (supra) } \\
\text { Artéria poplítea } \\
\text { Face }\end{array}$ & $\begin{array}{c}3-5 \\
3 \\
4-5 \\
4-5 \\
4-5 \\
4-5 \\
4 \\
1-4\end{array}$ & $\begin{array}{l}4 \\
1.3,4 \\
5 \\
5 \\
5 \\
5 \\
5 \\
2\end{array}$ \\
\hline D & Todas as outras & $1-2$ & 1 a 6 \\
\hline
\end{tabular}

Sua determinação depende da aplicação direta dos valores do ISS, RTS, idade do paciente e mecanismo de trauma (penetrante ou contuso), chegando-se diretamente à probabilidade de sobrevida (Ps), como mostrado na Tabela VIII.

Quanto à idade, o TRISS leva em consideração, se menor ou igual a cinquenta e quatro (54) anos (idade = zero) ou se maior de cinquenta e quatro (54) anos (idade = 1).

Estima a probabilidade de sobrevida (Ps) com um modelo logístico:

Ps $=1 /\left(1+\mathrm{e}^{-\mathrm{j}}\right)$

onde:

$$
\mathrm{J}=\mathrm{j}_{1}+\mathrm{j}_{2}(\mathrm{RTS})+\mathrm{j}_{3}(\text { ISS })+\mathrm{j}_{4}(\text { Idade })
$$

\begin{tabular}{|c|c|c|c|}
\hline \multirow{2}{*}{$\begin{array}{l}\text { Valores de A, B } \\
\text { ou C isolados }\end{array}$} & \multirow{2}{*}{\multicolumn{3}{|c|}{ Probabilidade de sobrevida (Ps) }} \\
\hline & A & & \\
\hline 5 & 0.84 & 0.93 & 0.96 \\
\hline 7 & 0.65 & 0.86 & 0.91 \\
\hline 9 & 0.42 & 0.71 & 0.78 \\
\hline 11 & 0.21 & 0.46 & 0.46 \\
\hline 13 & 0.09 & 0.21 & 0.14 \\
\hline 15 & 0.04 & 0.07 & 0.02 \\
\hline
\end{tabular}

TRAUMA AND INJURY SEVERITY SCORE (TRISS)

Este é um índice também proposto por Champion, em 1981, que procura associar o índice fisiológico (RTS) ao índice anatômico (ISS), levando em consideração, ainda, a idade e o mecanismo de trauma, se contuso ou penetrante ${ }^{(11)}$.

O TRISS é o método utilizado pelo Colégio Americano de Cirurgiões e tem fundamental importância na análise retrospectiva da probabilidade de sobrevida, sendo utilizado no $\operatorname{MTOS}^{(12)}$. A análise do TRISS permitirá avaliar a qualidade do serviço prestado na instituição, bem como compará-la com a de outros centros de trauma ${ }^{(12)}$.

\begin{tabular}{|c|c|c|c|c|}
\hline \multicolumn{5}{|c|}{ Tabela VIII - Pesos das constantes do TRISS } \\
\hline & $\mathrm{j}_{1}$ (constante) & $\mathrm{j}_{2}$ (RTS) & j3 (ISS) & j4 (idade) \\
\hline $\begin{array}{l}\text { Novos: } \\
\text { Contuso } \\
\text { Penetrante }\end{array}$ & $\begin{array}{l}-1.3054 \\
-1.8973 \\
\end{array}$ & $\begin{array}{l}0.9756 \\
1.0069 \\
\end{array}$ & $\begin{array}{l}-0.0807 \\
-0.0885\end{array}$ & $\begin{array}{l}-1.9829 \\
-1.1422 \\
\end{array}$ \\
\hline $\begin{array}{l}\text { Pesos (MTOS): } \\
\text { Contuso } \\
\text { Penetrante }\end{array}$ & $\begin{array}{l}-1.2470 \\
-0.6029 \\
\end{array}$ & $\begin{array}{l}0.9544 \\
1.1430 \\
\end{array}$ & $\begin{array}{l}-0.0768 \\
-0.1516\end{array}$ & $\begin{array}{l}-1.9052 \\
-2.6676 \\
\end{array}$ \\
\hline
\end{tabular}




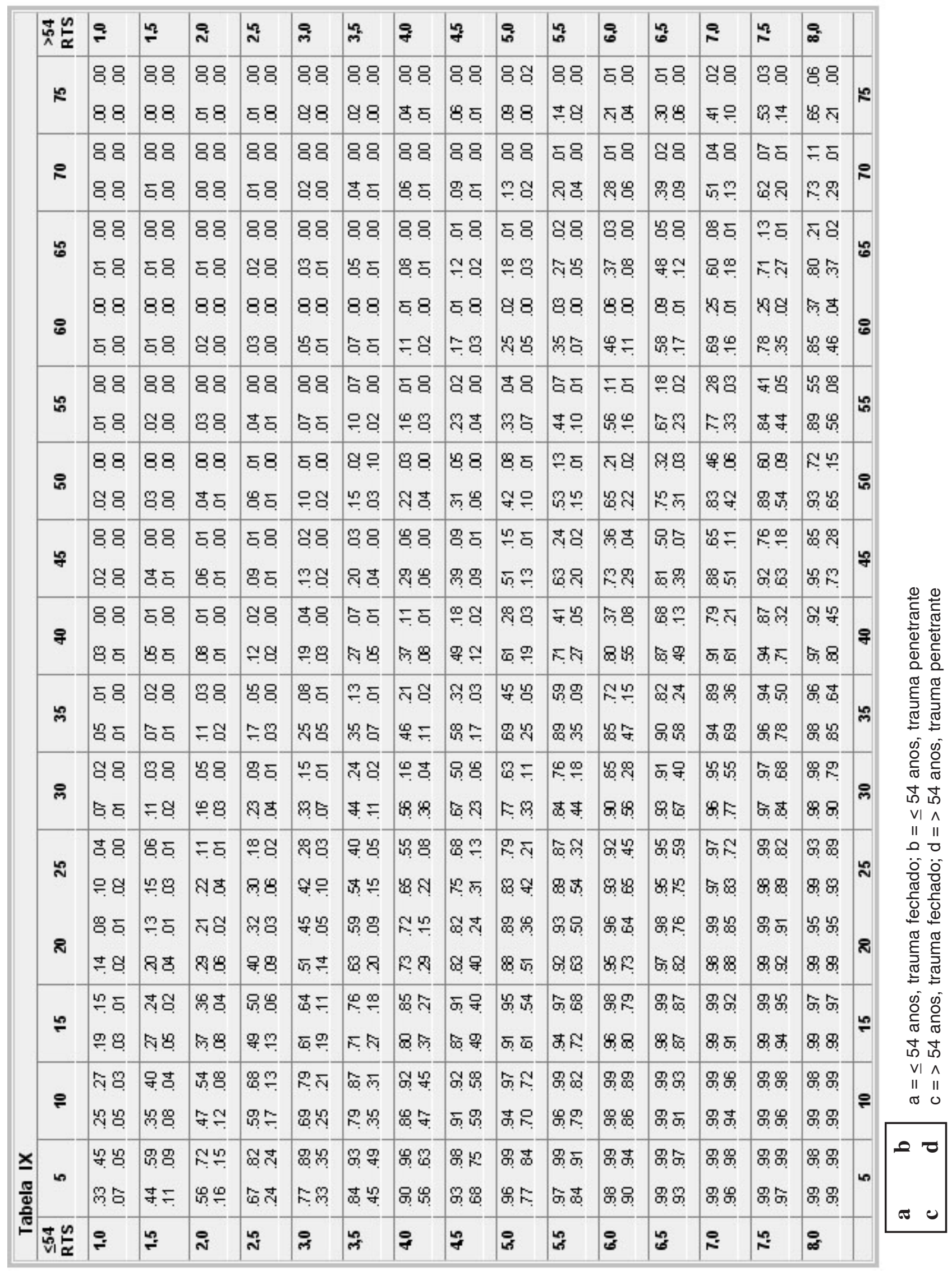


representa as combinações de valores do RTS e do ISS associadas com uma probabilidade de sobrevida de 50\%. Usando tabelas de PRE realizadas de acordo com as categorias de idade e mecanismo de trauma (penetrante ou contuso), as instituições podem definir as coordenadas de RTS e ISS para cada paciente nos eixos com Ps 50 apropriados ${ }^{(12)}$.

Tabela X - Exemplo de como se podem avaliar os casos, inferindo a qualidade do serviço prestado ao politraumatizado, através de sobreviventes inesperados (L's acima da linha) e não sobreviventes inesperados (D's abaixo da linha) distribuídos numa tabela que cruza os valores do ISS e RTS do paciente, situando abaixo ou acima da linha de probabilidade de $50 \%$ de sobrevida ou de mortalidade.

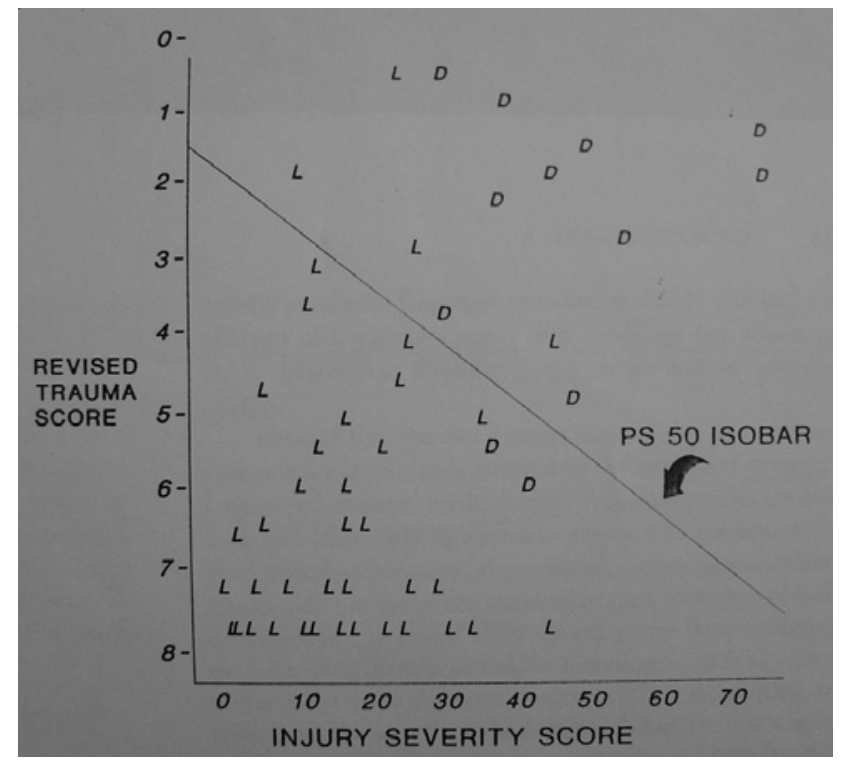

Cada L (live) acima da linha e D (death) abaixo da linha representa um paciente com resultados estatisticamente inesperados, cujo caso é merecedor de revisão clínica crítica. Um L acima da linha pode representar um triunfo terapêutico. Um D abaixo da linha, entretanto, pode refletir um dos seguintes situações: classificação inadequada do TRISS ou de seus índices componentes, erros no protocolo de tratamento, falha do sistema de atendimento ao traumatizado tais como atraso entre a lesão e seu tratamento ou o efeito de fatores não considerados no TRISS (ex.: condição do paciente previamente à lesão ou sequelas tardias, tais como embolia pulmonar) ${ }^{(13)}$. Esses resultados inesperados devem ser discutidos retrospectivamente por médicos, enfermeiras e auxiliares de enfermagem objetivando a análise crítica e correção dos erros identificados para que não mais ocorram ${ }^{(12)}$.

\section{PARTITION: contribuição dos cuidados pré-hos- pitalares}

É uma extensão do TRISS, que estima a contribuição dos cuidados pré-hospitalares para o potencial de sobrevida do paciente, separando os efeitos de tais cuidados com aqueles dos cuidados hospitalares ${ }^{(14)}$. É realizado, estimando-se a probabilidade de sobrevida (Ps), baseado em dados fisiológicos, tal como o tRTS, RTS na admissão, o ISS e a idade do paciente. Por exemplo, um primoroso atendimento pré-hospitalar pode ser complicado com pobres cuidados hospitalares, resultando em mortes evitáveis. Da mesma forma, um pobre cuidado pré-hospitalar pode ser compensado por um zeloso cuidado hospitalar, com melhora da sobrevida.

\section{ÍNDICE DE CARACTERIZAÇÃO DA GRAVI- DADE DO TRAUMA - A SEVERITY CHARACTERIZATION OF TRAUMA (ASCOT)}

O ASCOT foi desenvolvido para melhorar a capacidade de predição do TRISS, já que o TRISS não discrimina certos subtipos de politraumatizados, como ferimento penetrante por arma branca ou arma de fogo ou trauma contuso devido a acidentes automobilísticos ou quedas, e estes pacientes precisam ser considerados separadamente ${ }^{(15)}$. Consiste dos componentes AP, variáveis do RTS, mecanismo de trauma (se contuso ou penetrante) e uma refinada descrição da idade do paciente (tem uma classificação de cinco (05) pontos, em vez dos dois (02) usados no TRISS) (Tabela XI).

\begin{tabular}{|cc|}
\begin{tabular}{|c|} 
Tabela XI - Caracterização da idade do paciente \\
pelo ASCOT
\end{tabular} \\
\hline Valores & Idade (anos) \\
\hline 0 & $0-54$ \\
1 & $55-64$ \\
2 & $65-74$ \\
3 & $75-84$ \\
4 & $>85$ \\
\hline
\end{tabular}

O ASCOT é um índice mais discriminante e confiável de probabilidade de sobrevida do que o TRISS e os pacientes com prognósticos extremamente pobres ou excelente são excluídos (Tabela XII). Nesta tabela, os três primeiros correspondem a pacientes extremamente graves com lesões AIS $=6$ ou que 
chegaram à sala de admissão em parada cardiorrespiratória. O quarto tipo corresponde a pacientes com lesões leves, que não chegaram à sala de admissão em parada cardiorrespiratória.

Para os demais pacientes (não excluídos), os pesos do ASCOT para traumas contusos ou penetrantes derivam do seguinte modelo:

$$
\begin{aligned}
& \text { Ps }=1 /\left(1+\mathrm{e}^{-\mathrm{k}}\right) \\
& \text { onde: } \\
\mathrm{k}= & \mathrm{k}_{1}+\mathrm{k}_{2} \mathrm{ECG}+\mathrm{k}_{3} \mathrm{PAS}+\mathrm{k}_{4} \mathrm{FR}+\mathrm{k}_{5} \mathrm{~A}+\mathrm{k}_{6} \mathrm{~B}+\mathrm{k}_{7} \mathrm{C}+\mathrm{k}_{8} \\
& \quad \text { (Valor para idade) }
\end{aligned}
$$

O componente D foi excluído do modelo por não ser um contribuinte significante da mortalidade.

As constantes ponderadas do modelo ASCOT são mostradas na Tabela XIII.

Embora o RTS represente uma melhora substancial sobre seu precursor (Trauma Score), o ASCOT melhora a caracterização fisiológica, permitindo ponderar as variáveis do RTS (ECG, PAS e FR), diferenciando os pesos para traumas penetrantes ou contusos.

O TRISS e o ASCOT predizem melhor os resultados em pacientes com trauma penetrante do que nos com trauma contuso. Uma possível explicação é que a grande maioria dos traumas penetrantes são sub-

\begin{tabular}{|cc|}
\hline $\begin{array}{c}\text { Tabela XII - Pacientes excluídos da análise pelo } \\
\text { modelo ASCOT }\end{array}$ \\
\hline Excluídos & Descrição do paciente \\
\hline 1 & AIS $=6$, RTS $=0$ \\
2 & AIS $<6$, RTS $=0$ \\
3 & AIS $=6$, RTS $>0$ \\
4 & AIS $=1$ ou 2, RTS $>0$ \\
\hline
\end{tabular}

\begin{tabular}{|ccc|}
\hline \multicolumn{3}{|c|}{ Tabela XIII - Constantes do ASCOT } \\
\hline Variável & Contuso & Penetrante \\
\hline k1 & -1.1570 & -1.1350 \\
k2 & 0.7705 & 1.0626 \\
k3 & 0.6583 & 0.3638 \\
k4 & 0.2810 & 0.3332 \\
k5 & -0.3002 & -0.3702 \\
k6 & -0.1961 & -0.2053 \\
k7 & -0.2086 & -0.3188 \\
k8 & -0.6355 & -0.8365 \\
\hline
\end{tabular}

metidos a cirurgia, talvez facilitando uma descrição acurada, documentação e codificação de suas lesões.

Nesta tentativa de melhor caracterização das lesões, inferindo um prognóstico, há algumas limitações.

1) O estado fisiológico do paciente pode ser afetado por muitos fatores (ex.: tempo de lesão até a admissão, cuidados pré-hospitalares, presença de álcool ou drogas e a idade do paciente). Os pacientes mais jovens são capazes de compensar fisiologicamente grandes perdas da volemia até após seus sinais vitais serem assinalados. Para tais pacientes, os escores fisiológicos subestimam a gravidade. Drogas e álcool podem artificialmente deprimir os valores da Escala de Coma de Glasgow e superestimar a gravidade.

2) O diagnóstico anatômico, acurado e preciso, pode não ser sempre possível. As autópsias não são realizadas obrigatoriamente, o que permitiria adequada caracterização ${ }^{(16,17)}$. Além disso, muitas instituições não dispõem de métodos diagnósticos bioquímicos ou radiológicos, o que permitiria também melhor caracterização.

3) O processo de pontuação das lesões anatômicas é sujeito a variações por ele mesmo. A documentação das lesões pode ser inconsistente, especialmente se os médicos que fazem a caracterização não estiverem atentos para a classificação rigorosa do AIS. Por exemplo, as lacerações da veia cava inferior podem ser AIS 3 (se superficial), AIS 4 (profunda) e AIS 5 (se profunda, com perda de segmento). Assim, diferenças substanciais, no ISS ou no AP, podem resultar em variações na descrição clínica de lesões essencialmente iguais.

4) Variáveis não consideradas podem afetar o resultado, como uma doença subjacente, prévia ao trauma, levando a um maior tempo de internação, o que ocorre mais freqüentemente entre os idosos ${ }^{(18)}$

5) A falta de distinção entre mortes precoces e tardias. Trunkey descreveu a distribuição trimodal das mortes no trauma: $50 \%$ morrem na cena do acidente, $30 \%$ dentro das primeiras vinte e quatro (24) horas e $20 \%$ após o primeiro dia. Ele sugeriu que as mortes precoces são devidas à gravidade das lesões e seu efeito imediato, enquanto que as mortes tardias podem resultar de seqüelas como a má perfusão tecidual, sepse ou falência de múltiplos órgãos e sistemas.

6) Métodos são necessários para avaliar pacientes que chegam intubados ou em uso de medicações sedativas e bloqueadores neuromusculares, cuja Escala 
de Coma de Glasgow e a frequiência respiratória não podem ser avaliadas. Tais exclusões podem levar a sérias bias institucionais, pois, geralmente, estes pacientes excluídos têm taxa de mortalidade duas vezes maior que a dos pacientes inclusos.

\section{CONTROLE DE QUALIDADE}

Todo serviço de trauma deve rever seus resultados mensalmente. Os índices de gravidade são valiosos para identificar resultados aberrantes ou problemas potenciais no atendimento do politraumatizado, procurando uma solução de imediato. A revisão dos casos pode identificar mortes evitáveis e os fatores associados a esta situação é que devem ser revistos ${ }^{(19,20)}$.

As estratégias para melhora da qualidade no atendimento ao traumatizado deve envolver um plano de ação ou processo de diretrizes às vítimas de traumas, especialmente as de maior gravidade.

- Reênfase em certos princípios básicos dos cuidados com o traumatizado.

- Introdução ou ênfase das normas do ATLS.

- Ênfase nos protocolos de cuidados pré-hospitalares.

- Introdução de diretrizes para triagem inter-hospitalar.

- Introdução de diretrizes para cuidados de reabilitação.

- Introdução de diretrizes para transfusão sanguínea maciça.

- Introdução de normas institucionais para definir o momento de descontinuação das medidas de ressuscitação.

- Estabelecimento de normas para atendimento e tratamento de lesões traumáticas e queimaduras combinadas.

Um sistema de atendimento ao traumatizado, num âmbito regional, deve realizar revisões anuais de todas as fases do atendimento: pré-hospitalar, intrahospitalar, reabilitação e dados de autópsias dos pacientes vítimas de trauma, envolvendo os hospitais de todos os níveis de complexidade no atendimento ao traumatizado ( nível I, II e III). Deve avaliar, ainda, o impacto de alterações no sistema, como a introdução de programas de transporte aéreo, interrupção do uso de roupas pneumáticas antichoque, presença de desfibriladores nas ambulâncias, etc.

Adicionalmente, deve avaliar o valor de medidas preventivas, como uma mais rigorosa legislação em favor dos pedestres ou contra motoristas embriagados, obrigatoriedade do uso de cinto de segurança e proibição do fumo em áreas perigosas. Para isso, é essencial a adequada documentação das característi- cas epidemiológicas e demográficas dos traumas e delinear o impacto de medidas preventivas e estratégias terapêuticas.

Os hospitais dos diversos tipos de complexidade no atendimento ao traumatizado devem avaliar seus resultados de sobrevida/morte contra padrões nacionais e internacionais, sendo, para isso, essencial a adequada caracterização e registro das lesões, permitindo a utilização correta dos índices de gravidade. Tal avaliação deve incluir a análise do paciente após a alta hospitalar, como o retorno ao trabalho, incapacidade funcional e necessidade de reabilitação(21).

Tais registros são importantes, também, para traçar estratégias de redução nos custos do atendimento ao politraumatizado, quanto a recursos humanos, diagnósticos, monitorização e métodos terapêuticos, mantendo o mesmo alto padrão de qualidade ${ }^{(22)}$.

\section{OUTROS ÍNDICES DE TRAUMA}

Há vários outros índices de trauma que podem ser utilizados, dependendo das características próprias do serviço de trauma:

- Índice de trauma abdominal penetrante (Penetrating Abdominal Trauma Index - PATI $)^{(23,24)}$.

- Índice de trauma torácico penetrante (Penetrating Thoracic Trauma Index - PTTI $)^{(25)}$.

- Escore de trauma pediátrico (Pediatric Trauma Score - PTS) ${ }^{(26,27)}$

- Escore de gravidade do trauma geriátrico (Geriatric Trauma Severity Score - GTSS) ${ }^{(28)}$.

Há vários outros índices prognósticos, utilizados na prática médica diária, como: APACHE II e III (Acute Physiology And Chronic Health Evaluation) (29,30), MPM II (Mortality Probability Model) ${ }^{(31)}$, SAPS II (Simplified Acute Physiology Score) ${ }^{(32)}$, TISS (Therapeutic Intervention Scoring System) $(33,34)$, OSF (Organ-System Failure) $)^{(35)}$ e o MODS (Multiple Organ Disfunction System) ${ }^{(36)}$, porém não apresentam uma boa predição para os pacientes politraumatizados pela falta de um componente anatômico, que é essencial para avaliar a magnitude do trauma, sendo mais utilizados na Terapia Intensiva.

\section{ESTATÍSTICA Z}

A comparação entre a taxa de mortalidade de uma população (grupo de estudo) e aquela estimada pelo MTOS (Major Trauma Outcome Study) $)^{(3)}$, usando-se o método TRISS, pode ser obtida, usando-se a metodologia denominada “Z-statistic (37). 
Diversos aspectos devem ser levados em consideração na análise dos resultados obtidos com a estatística Z, especialmente quando as comparações entre o grupo de estudo e o MTOS não são estatisticamente significativas. Como o poder do teste estatístico é a habilidade de detectar a diferença estatística, é influenciado pelo tamanho da amostra ${ }^{(38)}$, pelo nível de significância escolhido, pela magnitude e direção da diferença (evolução observada, maior ou menor do que aquela esperada) e pela distribuição da probabilidade de sobrevida da população em estudo ${ }^{(39)}$

\section{ESTATÍSTICA W}

A estatística W é uma medida de significado clínico com relação à evolução, comparando o grupo em estudo e o grupo controle (MTOS) ${ }^{(12)}$. Representa a diferença entre o número de sobreviventes ou óbitos e o número estimado pelo MTOS, por cem doentes tratados ${ }^{(40)}$.

\section{CONSIDERAÇÕES FINAIS}

Os índices de trauma são instrumentos importantes para a aferição da qualidade da assistência médica prestada ao politraumatizado. Sua utilização, nos diversos hospitais que atendem pacientes vítimas de trauma, seja contuso ou penetrante, deveria ser rotineira.

Apesar de algumas limitações de cada índice, apresentam-se em constante evolução, numa tentativa de melhor definir a gravidade de cada paciente em particular, aumentando o seu poder de predição da sobrevida do politraumatizado.

Atualmente, o cálculo do Revisited Trauma Score (RTS), que pode ser feito logo na admissão do paciente na sala de politraumatizados, e do Injury
Severity Score (ISS), que deve ser feito após concluídos todos os diagnósticos do paciente por métodos de imagem ou mesmo cirurgias, deveria ser obrigatório. O cálculo destes dois índices, que, se correlacionados, mostra qual a probabilidade de sobrevida do paciente, através da tabela do Trauma and Injury Severity Score (TRISS), faz com que o médico preste mais atenção no seu cálculo e nos diversos fatores que podem afetar seu resultado, contrastando com o resultado final do tratamento do politraumatizado.

No aspecto individual de cada caso atendido, a revisão periódica de casos, inserindo-os no quadro do Preliminary Outcome-Based Evaluation (PRE), permite ao médico discutir sobre as intervenções que poderiam ter sido realizadas e em que momento, para um melhor resultado final.

Para o hospital ou mesmo para o sistema regional de atendimento ao traumatizado, o conjunto destes dados acumulados pode apontar falhas nas diversas fases do atendimento, indicando os pontos que deveriam ser reforçados ou melhor abordados.

O ASCOT (A Severity Characterization Of Trauma) representa um refinamento em relação ao ISS (Injury Severity Score), porém seu cálculo mais trabalhoso e a necessidade ainda, de uma maior quantidade de trabalhos para validar definitivamente seu papel fazem com que ele ainda não seja usado rotineiramente.

Para populações particulares, como as crianças e os idosos, o Pediatric Trauma Score (PTS) e o Geriatric Trauma Severity Score (GTSS), respectivamente, os fatores individuais destes pacientes, sejam eles pela imaturidade anatômica e fisiológica da criança e doenças concomitantes nos idosos, são levados em consideração no cálculo final destes índices, melhorando o seu poder de predição da sobrevida.

PEREIRA Jr. GA; SCARPELINI S; BASILE-FILHO A \& ANDRADE JI. Trauma severity indices. Medicina, Ribeirão Preto, 32: 237-250, july/sept. 1999.

ABSTRACT: Characterization and documentation of injury severity are requirements for the evaluation of trauma systems and development of iniciatives in injury control. Many indices employing measures of physiologic or anatomic derangement have been developed to quantify injury severity. Trauma scores represent na important and essential tool for clinical studies in trauma besides their use in the characterization of preventable deaths. This article describes several of the more commonly used indices and their applications.

UNITERMS: Trauma Severity Indices. Injury Severity Score. Abbreviated Injury Scale. Glasgow Coma Scale. Mortality. 


\section{REFERÊNCIAS BIBLIOGRÁFICAS}

1 - COIMBRA RSM; ANGLE N; SILVA SE; HOYT DB \& RASSLAN $S$. Índices de trauma: o que são e por que devem ser usados. Rev Col Bras Cir 24: 255-263, 1997.

2 - SHACKFORD SR; FRIDLUND PH; MCARDE M \& EASTMAN $A B$. Assuring quality in a trauma system - The medical audit committee: Composition, cost, and results. J Trauma 27: 866-875, 1987.

3 - CHAMPION HR; COPES WS; SACCO WJ; LAWNICK MM; KEAST SL \& FREY CS. The major trauma outcome study: establishing national norms for trauma care. J Trauma 30: 1356-1365, 1990.

4 - CHAMPION HR; SACCO WJ; COPES WS; GANN DS; GENNARELLI TA \& FLANAGAN ME. A revision of the trauma score. J Trauma 29: 623-629, 1989.

5 - CHAMPION HR; SACCO WJ; CARNAZZO AJ; COPES WS \& FOUTY WJ. Trauma score. Crit Care Med 9: 672-676, 1981.

6 - BAKER SP; O'NEILL B; HADDON JR W \& LONG WB. The injury severity score: a method for describing patients with multiple injuries and evaluating emergency care. J Trauma 14: 187 - 196, 1974.

7 - BAKER SP \& O'NEILL B. The injury severity score: an update. J Trauma 16: 882-885, 1976.

8 - THE ABBREVIATED INJURY SCALE - 1990. Revision. Association for the Advancement of Automotive Medicine, Des Plaines, Illinois, 1990.

9 - COPES WS; CHAMPION HR; SACCO WJ; LAWNICK MM \& BAIN LW. The Injury Severity Score revisited. J Trauma 29: $69-77,1988$.

10 - GENNARELLI TA; CHAMPION HR; SACCO WJ; COPES WS \& ALVES WM. Mortality of patients with head injury and extracranial treated in trauma centers. J Trauma 29: 1193-1201, 1989.

11 - CHAMPION HR; SACCO WJ \& COPES WS. Trauma Scoring. In: MOORE EE; MATTOX KL; FELICIANO DV, eds, Trauma, $3^{\text {a }}$ eds, Norwalk, Connecticut/San Mateo, Califórnia, Appleton \& Lange; cap. 5, p. 53-67, 1996.

12 - BOYD CR; TOLSON MA \& COPES WS. Evaluating trauma care: the TRISS method. J Trauma 27: 370-378, 1987.

13 - GILLOTAR; COPES WS; LANGAN E; NAJARIAN M; WISEMAN $J$ \& SACCO WJ. TRISS unexpected survivors - a statistical phenomen or a clinical reality? J Trauma 33: 743-748, 1992.

14 - SACCO WJ; JAMESON JW; COPES WS \& CHAMPION HR. PARTITION: a quantitative method for evaluating prehospital services for trauma patients. Comp Biol Med 18: 221-227, 1988.

15 - CHAMPION HR; COPES WS; SACCO WJ; LAWNICK MM; BAIN LW; GANN DS; GENNARELLI TA; MACKENZIE EJ \& SCHWAITZBERG S. A new characterization of injury. J Trauma 30: 539-546, 1990.
16 - HARVIEL JD; LANDSMAN I; GREENBERG A; COPES WS FLANAGAN ME \& CHAMPION HR. The effect of autopsy on Injury Severity and survival probability calculations. J Trauma 29: 766-773, 1989.

17 - BURKE MC; AGHABABIAN RV \& BLACKBOURNE B. Use of autopsy results in the emergency department quality assurance plan. Ann Emerg Med 19: 363-366, 1990.

18 - SACCO WJ; COPES WS; BAIN LW; MACKENZIE EJ; FREY CF; HOYT DB; WEIGELT JA \& CHAMPION HR. Effect of preinjury illness on trauma patient survival outcome. $\mathbf{J}$ Trauma 35: 538-543, 1993.

19 - CALES RH \& TRUNKEY DD. Preventable trauma deaths: a review of trauma care systems development. JAMA 254: 1059-1063, 1985.

20 - WILSON DS; MCELLIGOTT J \& FIELDING LP. Identification of preventable trauma deaths: confounded injuries? J Trauma 32: 45-51, 1992.

21 - DAVIS JW; HOYT DB; MCARDLE MS; MACKERSIE RC; EASTMAN B; VIRGILIO RW; COOPER G; HAMMILL F \& LYNCH FP. An analysis of errors causing morbidity and mortality in a trauma system: a guide for quality improvement. J Trauma 32: 660-666, 1992.

22 - SOUTHARD P. Trauma economics: suggestions for decreasing the cost of trauma care. J Emerg Nurs 19: 262-263, 1993.

23 - MOORE EE; DUNN EL; MOORE JB \& THOMPSON JS. Penetrating abdominal trauma index. J Trauma 21: 439-445, 1981.

24 - BORLASE BC; MOORE EE \& MOORE FA. The abdominal trauma index. A critical reassessment and validation. $\mathbf{J}$ Trauma 30: 1340-1344, 1990.

25 - IVATURY RR; NALLATHAMBI MN; STAHL WM \& ROHMAN M. Penetrating cardiac trauma. Quantifying the severity of anatomic and physiologic injury. Ann Surg 205: 61-66, 1987.

26 - TEPAS JJ; MOLLITT DL; TALBERT JL \& BRYANT M. Pediatric trauma score as a predictor of injury severity in the injured child. J Pediatr Surg 22: 14-18, 1987.

27 - KAUFMANN CR; MAIER RV; RIVARA FP \& CARRICO CJ. Evaluation of the pediatric trauma score. JAMA 263: 69-72, 1990.

28 - DEMARIA EJ; KENNEY PR; MERRIAM MA; CASANOVA LA \& GANN DS. Survival after trauma in geriatric patients. Ann Surg 206: 738-743, 1987.

29 - KNAUS WA; DRAPER EA; WAGNER DP \& ZIMMERMAN JE. APACHE II: a severity of disease classification system. Crit Care Med 13: 818-829, 1985.

30 - KNAUS WA; WAGNER DP; DRAPER EA; ZIMMERMAN JE; BERGNER M; BASTOS PG; SIRIO CA; MURPHY DJ; LOTRING T \& DAMIAN A. The APACHE III prognostic system. Chest 100: 1619-1636, 1991.

31 - LEMESHOW S; KHAR J; TERES D; AVRUNIN JS; GEHLBACH SH; RAPOPORT J \& RUE M. Mortality Probability Models $f$ or patients in the intensive care unit for 48 or 72 hours: a prospective, multicenter study. Crit Care Med 22: 1351-1358, 1994. 
32 - LE GALL JR; LEMESHOW S \& SAULNIER F. A new simplified acute physiology score (SAPS II) based on a European/ North American Multicenter Study. JAMA 270: 2957-2963, 1993.

33 - CULLEN DJ; CIVETTA JM; BRIGGS BA \& FERRARA LC. Therapeutic intervention scoring system: a method for quantitative comparison of patient care. Crit Care Med 2: 57-60, 1974.

34 - KEENE AR \& CULLEN DJ. Therapeutic intervention scoring system: update 1983. Crit Care Med 11: 1 - 3, 1983.

35 - KNAUS WA; DRAPER EA; WAGNER DP \& ZIMMERMAN JE. Prognosis in acute organ-system failure. Ann Surg 202: 685-693, 1985.

36 - MARSHALL JC; COOK DJ; CHRISTOU NV; BERNARD GR; SPRUNG CL \& SIBBALD WJ. Multiple organ disfunction score: a reliable descriptor of a complex clinical outcome. Crit Care Med 23: 1638-1652, 1995.
37 - FLORA JD. A method for comparing survival of burning patients to a standard survival curve. J Trauma 18: 701-705, 1978.

38 - TAYLOR MS; SACCO WJ \& CHAMPION HR. On the power of a method for comparing survival of trauma patients to a standard survival curve. Comput Biol Med 16: 1-6, 1986.

39 - COTTINGTONEM; SHUFFLEBARGER CM \& TOWSEND R. The power of the $Z$ statistic: Implications for trauma research and quality assurance review. J Trauma 29: 1500-1509, 1989.

40 - SACCO WJ; COPES WS; STAZ CF; SMITH Jr JS \& BUCKMAN Jr RF. Status of trauma patient management as measured by survival/death outcome: looking toward the $21^{\text {st }}$ century. J Trauma 36: 297-298, 1994.

Recebido para publicação em 11/08/99

Aprovado para publicação em 14/09/99 